\title{
A UPnP-based Decentralized Service Discovery Improved Algorithm
}

\author{
Yu Shi-cai*, Wu Yan-zhi, Guo Run-niu \\ School of Computer and Communication Lanzhou University of Technology, Lanzhou 730050, China \\ Troop 69050, Shawan 832100, China
}

\begin{abstract}
The current UPnP service discovery algorithm in the presence of the service can cause severe drops in the digital home network. The reason is that the root devices instantly send delay sending response messages and randomly selected independent response message congestion through simulation analysis. To solve these problems, an improved UPnP service discovery algorithm was given. Considering the length of the message and the bandwidth of the router, derived by testing the router the packet loss rate can be reduced.
\end{abstract}

Keywords: drop, service discovery, UPnP, queue size algorithm, best interval algorithm

\section{Introduction}

Digital Home [1] network refers to computer technology and network technology as the foundation, all kinds of home appliances through the different interconnection way communication and data exchange, realize household appliances between "the intercommunication", make people never leave home can be more convenient and quick access to information, thus greatly improve human living comfort and entertaining.

Home networks could consist of Information equipment, communications equipment, entertainment equipment, home electric digital home (Monitoring) device and electric hot water tables equipment, automation equipment, lighting equipment, security, home alarm for help equipment interconnection and management, as well as data and multimedia information sharing.

The main purpose of the interconnection equipment shared network services and resources, the remote call them. Service discovery protocol [2] can effectively solve the device how to automatically and effectively manage the problem of networked devices [3], a common choice UPnP service discovery algorithm [4]. However, with the service in the digital home network gradually increased UPnP service discovery algorithms appear inefficient.

Gribble [5] is trying to adopt a layered approach to solve the problem of Internet service discovery, this approach, however, ignores the "borderline" [6] of the home network environment. Chen Enyi et al [7] used in the UPnP root device (root devices) and the control point (control point) between a random delay is intermittently transmitted (RDCT) algorithms and scheduling delay instantaneous transmission (PDIT) algorithm, to some extent reduce the response to the news of the loss ratio, reducing the control point of the response message buffer size requirements. Ashish $\mathrm{T}$, et al [8] divide existing service discovery protocol into a "centralized" and "distributed", also achieved a certain effect on latency and network traffic performance in service discovery. On this basis, this paper focuses on distributed small network research, using the improved algorithm of the size of the queue (Queue size Algorithm) and the best interval (Best interval Algorithm) algorithm to solve the service discovery message latency and packet discard, in order to achieve a the service discovery smooth seamless connection.

\section{Distributed Service Discovery Algorithm}

In the distributed service discovery model, the network does not have a directory server, the user and the service provider before broadcast communications. In this research, the application layer through distributed computing to improve the rate of service discovery, the algorithm to be deployed in the active queue management (Active Queue Management, AQM), and the goal of the algorithm is: determine the router required transmission queue space and 
receive the next the outbreak of the news. This can be achieving by control the transmission rate of the service response. The proposed algorithm includes instructions and interpreted message queue length required and two consecutive outbreak interval equation, in order to avoid that the message will be discarded. OSI Open Systems Interconnection model, algorithm design at the application layer, protocol and application code, which may cause the news, broke to the network in terms of strategy. The next rule requires each computer routers send queue size or space, the router send queue and calculate the optimum interval must be available. The algorithm was test in the case of distributed routers.

\subsection{Queue Size Algorithm}

The algorithm used to calculate the size of the sending queue for each router, which is illustrated in Pseudo code Algorithm 1. The values $m$ and $n$ represent the number of clients and services connected to Routers ${ }^{R_{i}}$ respectively, where $i=1,2, \cdots$ Queue Size Algorithm.

For $\mathrm{i}=1$ to No. of routers

Start

If $\left(R_{i}\right.$ not connected to any node $)$

SQsize $_{\mathrm{Ri}}=\operatorname{Rim}+\operatorname{Rin}+1$.

End

\subsection{Best Interval Algorithm}

Assuming optionally the network topology can be divided into right and left halves. In pseudo-code algorithm 2, equation to ensure best interval allows specific router receive the next burst message before forwarding all messages to the destination (client). Further consider designated router queue sizes available, because it represents all the client (receiver) is connected to the router shared space between two or more continuous burst messages can be used to minimize the time required interval. Node $z$ is number candidate routers.

$\left(\mathrm{T}\left(\mathrm{x}_{\mathrm{k}}\right)\right)=\frac{\text { Message Size of service }}{k}$

In Equation (1), the value of $\left(\mathrm{T}\left(\mathrm{x}_{\mathrm{k}}\right)\right)$ represents the maximum of $\left(\operatorname{Time}\left(x_{k}\right)\right), k=1,2, \cdots .\left(\mathrm{T}\left(\mathrm{x}_{j}\right)\right)$ represents the link time of message connection.

Algorithm 2.pseudo code of interval algorithm

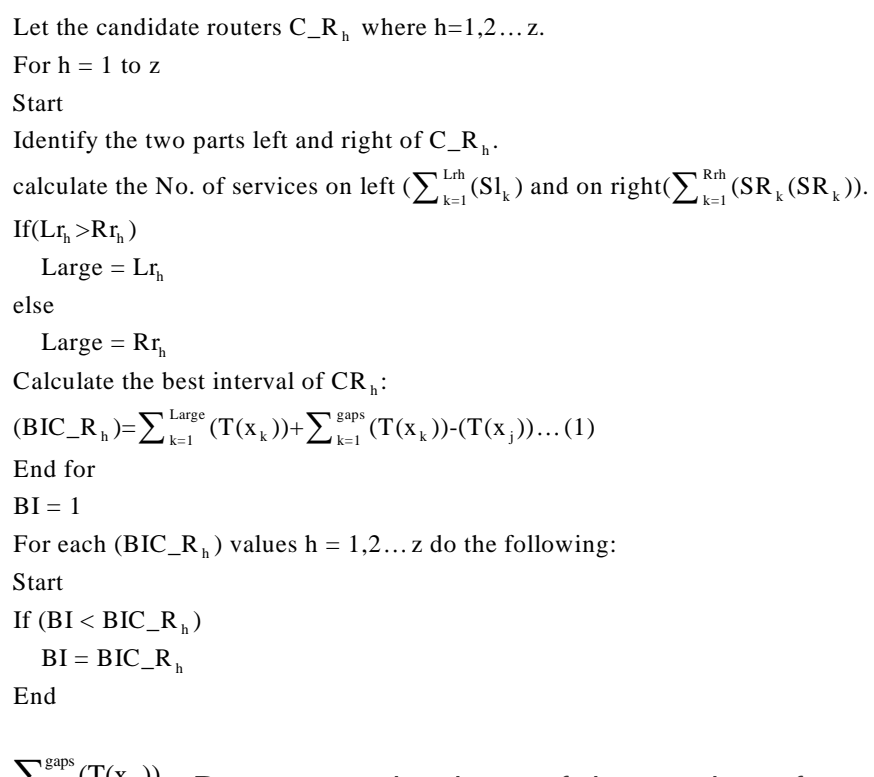

$\sum_{\mathrm{k}=1}^{\mathrm{gaps}}\left(\mathrm{T}\left(\mathrm{x}_{\mathrm{k}}\right)\right)$ : Represents the times of the number of message during the specific router does not receive messages from the nearest router. When there is a service connected directly to the nearest router, it would require at least two message times to reach the router.

IJEEI Vol. 1, No. 1, March 2013: $21-26$ 
The following equations represent best interval for any router:

$$
\left(\text { BIC_ } R_{h}\right)=\sum_{k=1}^{\text {Large }}\left(T\left(x_{k}\right)\right)+\sum_{k=1}^{\text {gaps }}\left(T\left(x_{k}\right)\right)-\left(T\left(x_{j}\right)\right) \ldots z \text {. }
$$

The identified queue value by the routers can be used to minimize the $\mathrm{BI}$ value. Overlapped space (OS) value represents this minimization. Pseudo code algorithm shows how to calculate the OS.

\section{Pseudo code 3. calculation steps of OS}

Identy the router neighbor to chosen router and this will be Rneightbour.

Let RLarge $=$ Rneighbour .

Let SQsize ${ }_{\text {RLarge }}=$ sending queue size of RLarge

Calculate OS $=\left(\right.$ SQsize $_{\text {RLarge }}-\mathrm{f}($ SRLarge $\left.)\right) / f\left(\mathrm{C}_{\mathrm{Ri}}\right) \ldots$ (2)

$\mathrm{f}($ SRlarge $)=\sum_{k=1}^{n}\left(S_{k}\right)$ represent the number of all services connected to Rlarge $_{\text {. }}$ $f\left(C_{R i}\right)=\sum_{k=1}^{m}\left(C_{k}\right)$ represent all the number of client connect to $R i$ 。S is measured from the number of messages. It represents all the empty messages space in the sending queue of the chosen router.

Equation (1)could use the OS value and be written as:

$$
(\mathrm{BI})=\sum_{\mathrm{k}=1}^{\mathrm{Big}} \mathrm{T}\left(\mathrm{x}_{\mathrm{k}}\right)+\sum_{\mathrm{k}=1}^{\mathrm{gaps}} \mathrm{T}\left(\mathrm{x}_{\mathrm{k}}\right)-\mathrm{T}\left(\mathrm{x}_{\mathrm{j}}\right)-\sum_{\mathrm{k}=1}^{\mathrm{OS}} \mathrm{T}\left(\mathrm{x}_{\mathrm{k}}\right)
$$

And now the question is, all the routers in the network must be evaluated in order to identify the best interval for the whole network? Which interval could be used for the network? The answer is: in a network, not all routers must be evaluated, but part of the candidate as evaluation, the longest interval will eventually be used, because logic use the longest interval will be avoided in all other routers on discarded news.

There are some conditions can help determine which routers in deciding on the best value interval for maximum impact.

\subsection{The Rules of Choosing Candidate Router}

The following rules indentify the bottlenecks of the messages flow paths. These rules required routers more time to forward the received messages during bursts:

(1) Identify the longest path between a client and service, and select the router which is connected to this client.

(2) Identify the router which connects the largest number to client and receives the largest number of services from another side of the network.

(3) Identify the router that located nearest the end of the network and is connected to one or more clients.

If all the routers connected to a client, all the routers which connect to the client must be selected in order to compare between two consecutive burst of messages that reach these routers consecutively.

In the case of two (or more) consecutive bursts of the message is sent to the same client, and the client is separately connected to the router, which means that there are two (or more) receiver logic connected to router, which should be in the calculation (OS) of the value when considering.

A client may meet more than one of the previous conditions, in other words, having the longest path and the client may be the same client, is connected to a router, which receives the maximum number of services, it does not cause any problems. All candidate routers must be evaluated, the longest time interval is the best time interval in a network, it can ensure that no lost messages. 


\section{The Results Of Simulation}

The application of simulation models clarify AQM on performing of UPnP. This was used to compare the algorithm, and under normal circumstances UPnP. Assume that realistic application scenarios network design includes four router (R0, R1, R2 and R3), in which each router connected to the three services (S0, S1, ..., and S9). Except R2 connected to six clients (C0, C1... C5). The network parameters are given in Table 1.

(1) (CO, C1 ... C5) send multicast information on the network to discover all services.

(2) The client sends a response message for all services requested. Algorithm, each service separately any consecutive reply message within a certain period of time. However, under normal circumstances, the service reply to the discovery request dependency.

There are a UDP background traffic (S0, S8) and (S1 the S7), S0, S1 is connected to the R0, S7 and S8 connected to R3. The backwards flow rate is 0.01 and the message size is different.

Routing rules based on the selected candidate, R2 is a candidate routers. Obviously, the service connect to R6 has the longest path to reach the $\mathrm{C} 0$ and $\mathrm{C} 1$.

Table 1. Network Parameters

\begin{tabular}{ll}
\hline Parameter name & Value \\
\hline Bandwidth among routers (Main links) & $512 \mathrm{~Kb}$ \\
Bandwidth between routers and other nodes (Sub links) & $256 \mathrm{~Kb}$ \\
Delay in main and sub & Oms \\
Queue Type & Drop Tail \\
Routing Protocol & DSDV \\
Message Length of discovery(Multicast) & 64 bytes \\
Message Length of discovery reply(Unicast) & 128 bytes \\
Message Length of back ward traffic & $100,200,300 b y t e s$ \\
Simulation & 100.0 seconds \\
\hline
\end{tabular}

According to the size of the queue algorithm, the queue size for all the routers except $\mathrm{R} 2$ is:

SQsize $_{R 0}=4+1=5$ packets , while $R 2=2+1=3$ packets

In order to calculate the best interval, Overlapped Space(OS) must be calculated.

OS $=(5-3) / 4=0.5$ messages spaces.

All replying messages are different in the link of bandwidth and equal in sizes:

$T\left(x_{k}\right)$ could be two values.

$T\left(x_{k}\right)=\frac{256 * 8}{512 * 1024} \approx 0.004 s^{\prime} 1-T\left(x_{k}\right)=\frac{256 * 8}{1000 * 1024}=0.002 s$

The Best Interval:

$(B I)=\sum_{k=1}^{12} 0.004+\sum_{k=1}^{1} 0.004-0.004-\sum_{k=1}^{0} 0.004=0.048 s$.

The flowing Figure 1 and Figure 2 show the network utilization in algorithm and normal cases when this interval is applied in simulator.

Growth enough to receive burst in the next message is to forward all of the sudden news when the news broke, the major part of the network utilization is improved. Although the algorithm is to make the calculation, in the case of using the algorithm to reach the upper limit of network utilization will result in the loss of messages. This is because the algorithm does not take into account the factors of the backward message.

IJEEI Vol. 1, No. 1, March 2013 : 21 - 26 


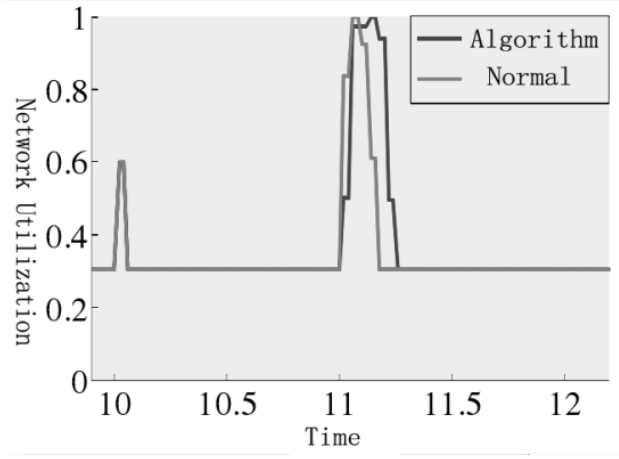

Figure 1. Network Utilization Comparison (Main Link)

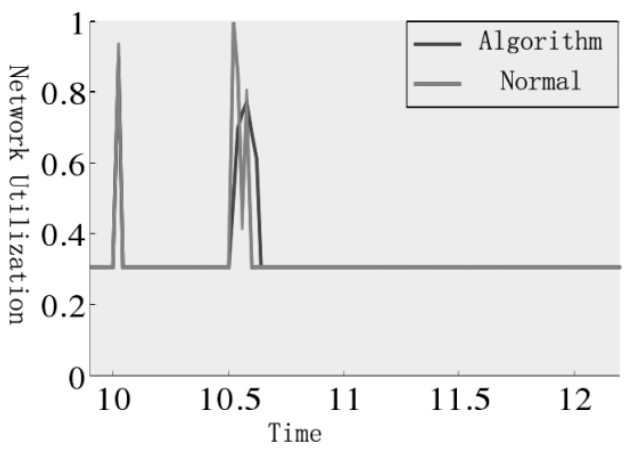

Figure 2. Network Utilization Comparison (Sub Link)

The sub Link results show that, under normal circumstances, there are obvious differences, between normal case and using an algorithm, as is shown in Figure 2. Irrespective of active queue management in the utilization of the network in the normal case to reach the upper limit and discard the message, while in the case of using an algorithm, the network utilization is less than $85 \%$ and not discarded messages.

The previous figures show that the algorithm reduces discovery time by sending rate to avoid message loss. This ensures that all messages to their destination, without loss of speed and efficiency. In addition, the figure also shows the impact of the backward transport network utilization and lost messages, these must be considered in the algorithm to improve its performance.

Figure 3 shows comparison AQM and under normal cases of the discovery rate. Overall, the service discovery algorithm uses a higher rate than normal case. In the case of using an algorithm, the detection rate is between $77 \%-100 \%$, and under normal cases, found $55 \%-88.88 \%$.

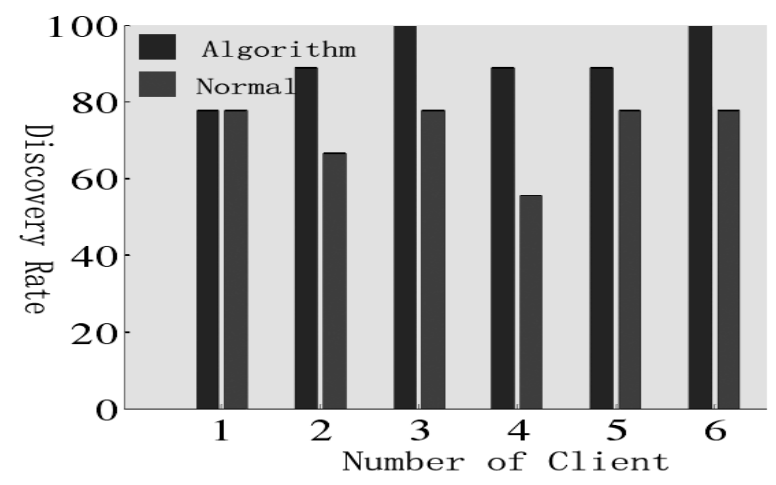

Figure 3. Discovery Rate Comparison

\section{Conclusion}

During service discovery protocols, the router dropping messages results in incomplete discovery process, which causes delay and inefficiency in connected nodes(s) performance. This algorithm solved this problem. This algorithm introduces a new method to the time interval required to determine the relationship between the available spaces twice broke the news in the router queue. This indicates that the specific method to calculate the required transmit queue size. These methods can easily be modified to reduce the best interval by increasing the size of the queue and compete and vice versa. This algorithm take the bandwidth of the network 
configuration and size of the message in into consideration, thus affecting the rate of the transmission and reception.

Previous experimental results show that the improved network utilization discovery rate. In addition, these Figures also show that the impact of the algorithm, the ratio of consumption of network resources, which indicates that also need to provide the additional different parameters, the proposed algorithm available network resources, in order to cope with changing in the available network.

\section{References}

[1] Yiakoumis Y, Huang T, Yap K, et al. Putting home users in charge of their network. Systems and Infrastructure for the Digital Home, ACM Ubicomp workshop. 2012; 9: 1-6.

[2] AIMejibli, Colley. Evaluating Transmission Time of Service Discovery Protocols by using NS2 Simulator. Wireless Advanced (WiAD). 2010; 11: 13-17.

[3] Intisar, Al-Mejibli, Martin, Colley. Enhancing Service Discovery Performance over Home networks. Computer Networks \& Communications (IJCNC). 2012; 4(2): 85-100.

[4] UPnP[EB/OL]. http://baike.baidu.com/view/27925.htm.

[5] Gribble SD, Welsh M, Behren RV, et al. The Ninja architecture for robust Internet-scale systems and services. Computer Networks. 2001; 35(4): 473-497.

[6] Kind berg T, Fox A. System software for ubiquitous computing. Pervasive Computing. 2002; 1( 1): 7081.

[7] Chen Enyi, SHI Yuanchun, Xu Guang. UPnP service discovery algorithm performance analysis and improvement. Journal of Tsinghua University. 2006; 46(4): 600-608.

[8] A shish T Bhole, Shital Ranade. Approach of Association Rules Mining for Service Discovery in Mobile Adhoc Network. International Journal of Computer Applications. 2012; 44(8): 37-42. 